Please do not remove this page

RMIT

UNIVERSITY

\title{
Polymer long-period raised rib waveguide gratings using nano-imprint lithography
}

Perentos, Nicholas; Kostovski, Gorgi; Mitchell, Arnan

https://researchrepository.rmit.edu.au/esploro/outputs/9921861950001341/filesAndLinks?institution=61RMIT_INST\&index=null

Perentos, N., Kostovski, G., \& Mitchell, A. (2005). Polymer long-period raised rib waveguide gratings using nano-imprint lithography. IEEE Photonics Technology Letters, 17, 2595-2597.

https://doi.org/10.1109/lpt.2005.859130

Document Version: Published Version

Published Version: https://doi.org/10.1109//pt.2005.859130

Repository homepage: https://researchrepository.rmit.edu.au

(c) 2005 IEEE. Personal use of this material is permitted. However, permission to reprint/republish this material for advertising or promotional purposes or for creating new collective works for resale or redistribution to servers or lists, or to reuse any copyrighted component of this work in other works must be obtained from the IEEE.'

Downloaded On 2023/04/26 22:29:17 +1000 


\title{
Polymer Long-Period Raised Rib Waveguide Gratings Using Nano-Imprint Lithography
}

\author{
Andreas Perentos, Gorgi Kostovski, and Arnan Mitchell
}

\begin{abstract}
This letter presents the fabrication and demonstration of a long-period raised rib waveguide grating using nano-imprint lithography. The device consists of a lower UV15 cladding, where relief-gratings are implemented, and an NOA73 raised rib core waveguide. Spectral transmission reveals a resonance at $1585 \mathrm{~nm}$ with about $10-\mathrm{dB}$ rejection and 12-nm linewidth.
\end{abstract}

Index Terms-Long-period waveguide gratings (LPWGs), nanoimprint lithography (NIL), optical polymers, optical waveguide filters, polydimethylsiloxane (PDMS), soft lithography.

\section{INTRODUCTION}

$\mathbf{L}$ ONG-PERIOD fiber gratings are popular devices used as band-rejection filters [1], sensors [2], erbium-doped fiber amplifier flatteners [3], and dispersion compensators [4]. Their simple geometry and very modest resolution requirements make them good candidates for low-cost photonic applications such as coarse wavelength-division multiplexing. The mass manufacture of these gratings is, however, limited by the serial nature of optical fiber. To enable mass manufacture of multiple units in parallel, it would be advantageous to realize long-period waveguide gratings (LPWGs) on planar substrates.

Planar LPWGs have been proposed [5] and demonstrated in epoxy-clad ion-exchanged BK7 glass slab waveguides [6] and epoxy-clad benzocyclobutene ridge (channel) waveguides [7]. The relief gratings in both devices were formed using reactive ion etching. Benzocyclobutene ridge waveguide gratings have also been fabricated using a $\mathrm{KrF}$ excimer laser [8]. The performance of these devices is good as the rejection at resonance is strong (15-25 dB depending on polarisarion), they can be thermally tuned, are insensitive to polarization, and have reasonably narrow 3-dB bandwidth (approximately $10 \mathrm{~nm}$ ). The fabrication process though involves several steps and the uniform etching of the grating is particularly crucial. An alternative simpler fabrication process would be of great interest.

In this letter, we report on the use of nano-imprint lithography (NIL) techniques to fabricate long-period raised-rib waveguide gratings in optical polymer materials. This technique was pioneered by Xia and Whitesides [9] and has been recently used for fabricating polymer microring resonators [10] and electrooptic polymer Mach-Zehnder modulators [11]. It is shown that this technique is rapid and straight-forward and can realize planar

Manuscript received July 25, 2005; revised August 14, 2005.

The authors are with the Microelectronics and Materials Technology Centre (MMTC), School of Electrical and Computer Engineering, RMIT University, Melbourne VIC 3001, Australia (e-mail: s9812291@student.rmit.edu.au; s9909628@student.rmit.edu.au; arnan.mitchell@rmit.edu.au).

Digital Object Identifier 10.1109/LPT.2005.859130

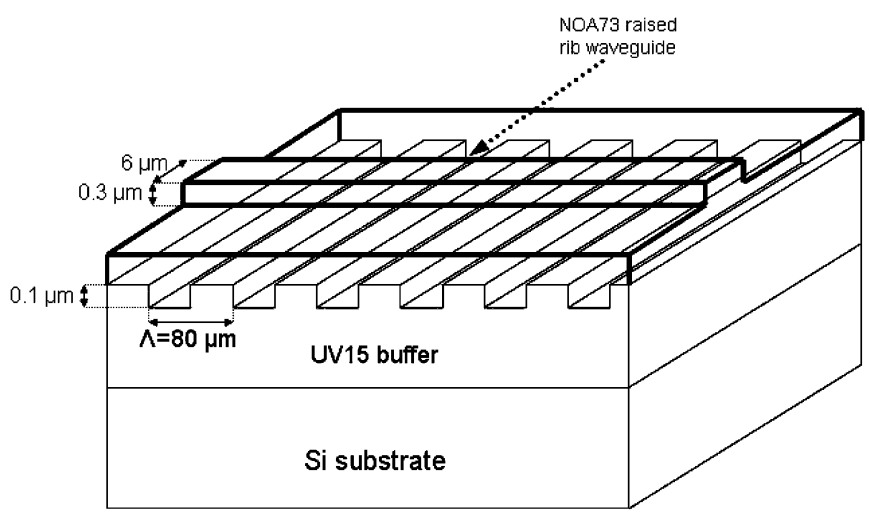

Fig. 1. Cross section of long-period raised rib waveguide with grating on the cladding-core interface.

LPWGs with similar performance to those reported previously [6]-[8].

\section{Device Design And Basic Modeling}

The cross section of the proposed device is shown in Fig. 1. Silicon is used as a substrate due to the fact that it is readily available and is exceptionally flat. Silicon has a very high refractive index $(n \sim 3.5$ at $1550 \mathrm{~nm})$ and this can cause leakage of optical power from the guided modes. To minimize leakage, a relatively thick $(20 \mu \mathrm{m})$ buffer layer of low index UV15 polymer ( $n \sim 1.508$ at $1550 \mathrm{~nm}$ ) must be inserted between the waveguide and the silicon substrate.

The core layer is composed of a higher index NOA73 polymer $(n \sim 1.5368$ at $1550 \mathrm{~nm}$ ) and is approximately $3.5 \mu \mathrm{m}$ thick providing single-mode vertical confinement. Lateral confinement is achieved using a raised rib structure formed on the top side of this layer. The waveguide is not capped and, thus, the top cladding is air.

The grating is implemented on the interface between the cladding and the core. The grating period $\wedge$ can be calculated using the phase matching condition [1] and is equal to $\lambda /\left(n_{\mathrm{eff}, 1}-n_{\mathrm{eff}, 2}\right)$, where $\lambda$ is the resonant wavelength and $n_{\mathrm{eff}, 1}$ and $n_{\mathrm{eff}, 2}$ are the effective refractive indexes of the fundamental core mode and a forward-propagating cladding mode. The individual effective indexes of propagation are extremely sensitive to the thicknesses of the two layers. This makes the resonance wavelength similarly sensitive and, therefore, hard to predict and demonstrate precisely in practice as exact thicknesses are not easily achieved. One way of accounting for this is by varying slightly the core thickness. The grating depth is selected so that that maximum coupling can be achieved 


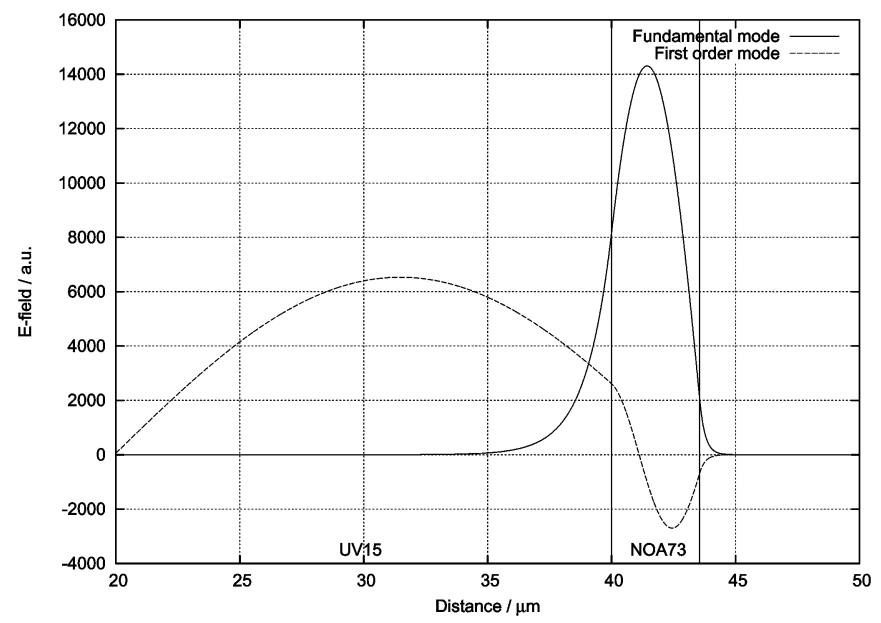

Fig. 2. Normalized fundamental NOA73 guided core mode and first-order forward-propagating UV15 cladding mode. The grating is implemented on the UV15-NOA73 interface.

at a certain propagation length and, at the same time, grating radiation losses are minimized.

The propagation characteristics of the raised rib waveguide, shown in Fig. 1, were modeled using a one-dimensional finitedifference mode solver and the effective index method at transverse-electric (TE) polarization. This analysis is only an approximation as the actual core would be composed of raised rib waveguides of $6 \mu \mathrm{m}$ in width and $0.3 \mu \mathrm{m}$ in depth. The UV15 layer was set to $20 \mu \mathrm{m}$ thick and the NOA73 layer at $\sim 3.55 \mu \mathrm{m}$. Their bulk refractive indexes, measured previously with a Metricon prism coupler at TE polarization, are 1.5080 and 1.5368 in the vicinity of $1550 \mathrm{~nm}$.

The effective index analysis indicated that the rib waveguide was single-moded. Fig. 2 presents the first two eigenmode solutions at a cross section through the center of the rib. The fundamental mode is guided in the NOA73 while the first-order mode resides primarily in the cladding. We select a grating period to couple the fundamental mode and the first-order mode. Their respective effective indexes for TE polarization at $1585 \mathrm{~nm}$ are roughly calculated to be 1.527 and 1.508 . Thus, a grating period of $80 \mu \mathrm{m}$ is required to observe a resonance in the vicinity of $1585 \mathrm{~nm}$. As the final fabricated UV15 and NOA73 thicknesses will most likely have some degree of variation to the target ones, a range of NOA73 thicknesses from 3.5-3.7 $\mu \mathrm{m}$ would be implemented to account for this. If the grating is made $0.1 \mu \mathrm{m}$ deep, the length required to achieve full transfer of power from one mode to another, known as the coupling length, would be $3.29 \mathrm{~mm}$. Over a 10-mm-long device with gratings of period 80 and $0.1 \mu \mathrm{m}$ in depth, the coupling efficiency is estimated at $99.6 \%$. Planar LPWG with similar index contrast and grating depth have been demonstrated with acceptable radiation losses [6]. The expected linewidth can be calculated as $0.8 \lambda^{2} /(\Delta n L)[1]$. Thus, using the parameters above, a 1-cm-long grating would provide a linewidth of approximately $10 \mathrm{~nm}$.

\section{FABRICATION}

The process used to fabricate the planar LPWG devices is illustrated in Fig. 3. Si wafers $\left(3^{\prime \prime}\right)$ of 100 orientation were

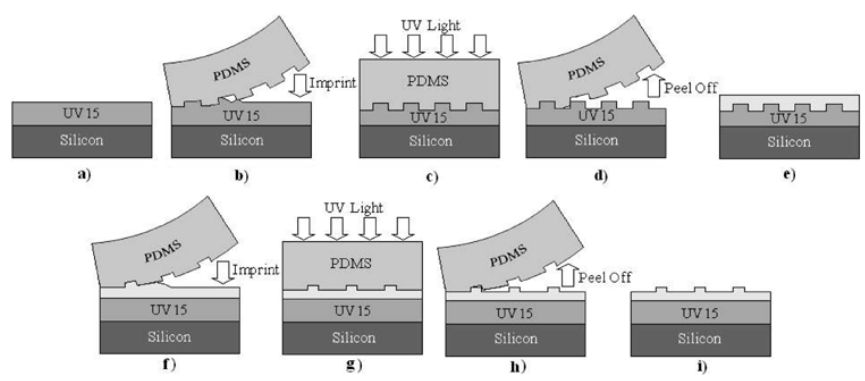

Fig. 3. Fabrication technique illustrating successive imprinting steps.
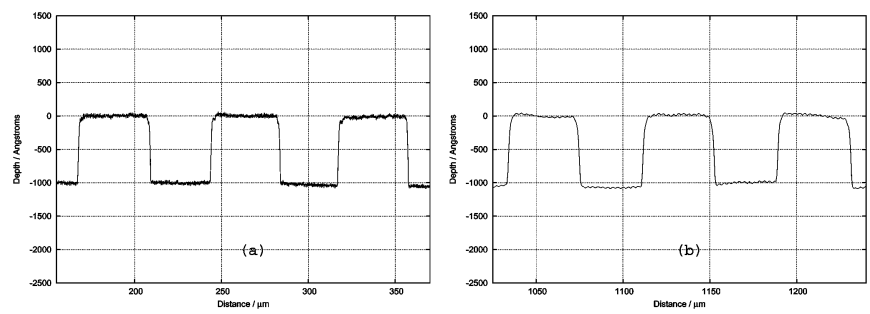

Fig. 4. Profiles of (a) $0.1-\mu$ m-deep Ti grating master and (b) imprinted UV15 grating replica. The resulting grating profile matches very well the profile of the Ti master.

used as substrates. The UV15 cladding layer was spin coated at $1000 \mathrm{rpm}$ yielding the $20-\mu \mathrm{m}$-thick layer [Fig. 3(a)]. An $80-\mu \mathrm{m}$ period grating mold composed of polydimethylsiloxane (PDMS) was created from a $0.1-\mu \mathrm{m}$-deep Ti master. The mold is gently applied to the uncured UV15 layer laterally with a slight bend [Fig. 3(b)]. This is one of the great advantages of PDMS as it is extremely flexible and can replicate structures with very high accuracy. It is then cured by exposure to a $365-\mathrm{nm}$ mercury UV lamp of $7.5 \mathrm{~mW} / \mathrm{cm}^{2}$ intensity for $5 \mathrm{~min}$ [Fig. 3(c)]. The NOA73 layer is then spin coated at various neighboring speeds (4000-4500 rpm) yielding 3.5-3.7- $\mu \mathrm{m}$-thick layers [Fig. 3(e)]. This is an essential step as it accounts for the fabrication inaccuracies of expected spin coating thickness and imprinting induced thickness modifications for both the gratings and subsequent ribs. This makes the resonance observation easier. Another PDMS mold is used to imprint raised rib waveguides of $6 \mu \mathrm{m}$ in width and $0.3 \mu \mathrm{m}$ in depth [Fig. 3(f)-(i)]. This time, the NOA73 layer is cured for $20 \mathrm{~min}$ in bursts of $5 \mathrm{~min}$ as it takes longer to harden.

To demonstrate the replication quality of the NIL process, an AMBIOS XP-2 profilometer was used to profile the $80-\mu \mathrm{m}$ period grating Ti master, from which the PDMS mold was taken, and the imprinted grating pattern on the UV15 cladding. The profiles are presented in Fig. 4(a) and (b), respectively. The replication is sufficiently accurate for this LPWG application. Some regions were not as uniform as the ones displayed in Fig. 4(b). This could be attributed to the fact that the same PDMS grating mold was used for several imprints, hence introducing in some regions some polymer residue. Also, the imprinting process displaces, to a degree, the associated layer and, hence, induces an overall slight thickness modification. The period and depth of the grating though remain unaltered.

An advantage to using optical polymers for waveguides is that excellent end-faces (for butt coupling) can be produced by simply dicing using a high-speed automatic polishing saw 


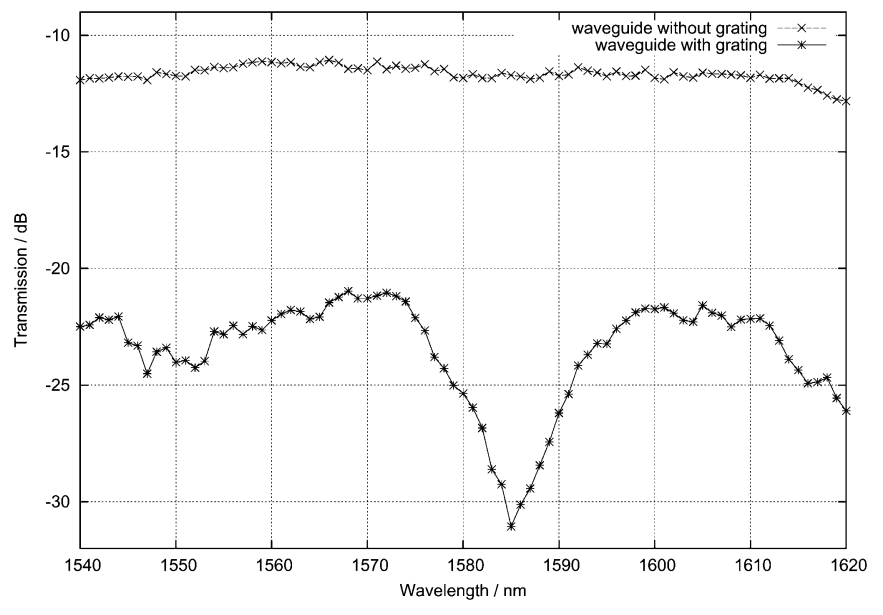

Fig. 5. Normalized spectral transmission of long-period raised rib waveguide grating for TE polarization. The normalized response of an identical rib without the grating is also plotted to distinguish between grating loss and polymer transmission loss.

(disco DAD 321). The samples were prepared for optical testing using this approach.

\section{RESULTS}

The transmission spectrum of the device with an NOA73 nominal thickness of $3.7 \mu \mathrm{m}$, at room temperature, through an $80-\mu \mathrm{m}$ period grating and $6-\mu \mathrm{m}$-wide raised rib waveguide was measured via butt coupling with an Agilent HP tunable laser and a Newport power meter, both driven form a LabView interface. The normalized transmission spectrum for TE polarization is shown in Fig. 5. The transmission spectrum of an identical waveguide without the grating is also plotted for loss comparison. A resonance is observed at $1585 \mathrm{~nm}$ with a rejection level of $\sim 10 \mathrm{~dB}$ and a $3-\mathrm{dB}$ bandwidth of $\sim 12 \mathrm{~nm}$. Each characteristic is close to its expected value.

\section{DisCuSSION}

Slight differences in the extinction and linewidth could be attributed to approximations made with the effective index model. In particular, lateral radiation of the first-order mode has not been accounted for. More rigorous modeling could be achieved using a wide angle beam propagation method. Fluctuations on both sides of the rejection band are attributed to nonuniform grating regions caused by polymer residue on the PDMS mask during imprinting. The overall optical insertion loss of the device is relatively high due to polymer material absorption and also modal mismatch between the fiber and the raised rib geometry. This could be remedied using perfluorinated polymers and optimizing the raised rib waveguide geometry. The loss attributed to the presence of the grating is approximately $10 \mathrm{~dB}$ and could be reduced by implementing shallower gratings with greater uniformity. Investigations on the use of temperature to tune the grating are underway. The use of a liquid superstrate for tuning is also being explored.

\section{CONCLUSION}

We have successfully fabricated a raised rib polymer LPWG using PDMS NIL. The grating is implemented on the lower UV15 cladding layer at its interface with the NOA73 core layer. Resonance is demonstrated at $1585 \mathrm{~nm}$ with a rejection level of $10 \mathrm{~dB}$ ( $\sim 90 \%$ coupling efficiency) and a 3-dB bandwidth of $12 \mathrm{~nm}$. This technique is fast and simple and could be applied on many other optical polymers.

\section{REFERENCES}

[1] A. M. Vengsarkar, P. J. Lemaire, J. B. Judkins, V. Bhatia, T. Erdogan, and J. E. Sipe, "Long-period fiber gratings as band-rejection filters," $J$. Lightw. Technol., vol. 14, no. 1, pp. 58-65, Jan. 1996.

[2] V. Bhatia, "Applications of long-period gratings to single and multi-parameter sensing," Opt. Express, vol. 4, pp. 457-466, 1999.

[3] P. F. Wysocki, J. B. Judkins, R. P. Espindola, M. Andrejco, and A. M. Vengsarkar, "Broad-band erbium-doped fiber amplifier flattened beyond $40 \mathrm{~nm}$ using long-period grating filter," IEEE Photon. Technol. Lett., vol. 9, no. 10, pp. 1343-1345, Oct. 1997.

[4] M. Das and K. Thyagarajan, "Dispersion compensation in transmission using uniform long period fiber gratings," Opt. Coтmun., vol. 190, pp. 159-163, 2001.

[5] V. Rastogi and K. S. Chiang, "Long-Period gratings in planar optical waveguides," Appl. Opt., vol. 41, pp. 6351-6355, 2002.

[6] K. S. Chiang, K. P. Lor, C. K. Chow, H. P. Chan, V. Rastogi, and Y. M. Chu, "Widely tunable long-period gratings fabricated in polymer-clad ion-exchanged glass waveguides," IEEE Photon. Technol. Lett., vol. 15, no. 8, pp. 1094-1096, Aug. 2003.

[7] K. S. Chiang, C. K. Chow, H. P. Chan, Q. Liu, and K. P. Lor, "Widely tunable polymer long-period waveguide grating with polarization-insensitive resonance wavelength," Electron. Lett., vol. 40, pp. 422-424, 2004.

[8] K. P. Lor, Q. Liu, and K. S. Chiang, "UV-written long-period gratings on polymer waveguides," IEEE Photon. Technol. Lett., vol. 17, no. 3, pp. 594-596, Mar. 2005

[9] G. M. W. Y. Xia, "Soft lithography," Angewandte Chemie Int. Edition, vol. 37, pp. 550-575, 1998

[10] J. K. S. Poon, Y. Huang, G. T. Paloczi, and A. Yariv, "Soft lithography replica molding of critically coupled polymer microring resonators," IEEE Photon. Technol. Lett., vol. 16, no. 11, pp. 2496-2498, Nov. 2004.

[11] G. T. Paloczi, Y. Huang, A. Yariv, J. Luo, and A. K.-Y. Jen, "Replicamolded electro-optic polymer Mach-Zehnder modulator," Appl. Phys. Lett., vol. 85, pp. 1662-1664, 2004. 